\title{
Microscopic Pore Structural Characteristics in Coal Particles
}

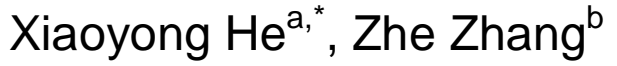 \\ CCTEG Shengyang Research Institute, Shenyang 110016, P.R. China \\ aemail:1220931431@qq.com, bemail:cumtblxf@163.com
}

Keywords: Pore, Microscopic structure, Coal particle, Adsorption isotherm

\begin{abstract}
In order to investigate the internal pore structure of coal, the adsorption isotherms of coal particles with different sizes were measured using the Quantachrome Autosorb - iQ specific surface area and pore size analyzer. The pore characteristic parameters obtained were analyzed including the specific surface area, pore volume and average pore diameter. The use of low pressure nitrogen adsorption allowed us to gain clearer insight into the structural characteristics of pores in the coal body. The results indicate that the adsorption isotherms of coal particles with different sizes displayed a similar variation trend. The specific surface area and total pore volume of coal particles decreased gradually with increasing particle size. Whereas, the average pore size increased with the rise of particle size. The results can provide theoretical foundation for studying the gas adsorption, desorption and seepage flow mechanism of coal.
\end{abstract}

\section{Introduction}

Coal is a kind of typical dual-porous medium pore and fracture[1]. The porosity of coal depends on the structure characteristics of coal. At the same time, the pore characteristics of coal is largely determines the properties of coal, such as the capacity of gas adsorption, coal's permeability and strength[2,3].Research has proved that pore in the coal is made up of different orders of magnitude of porosity which is the small diameter from $0.5 \mathrm{~nm}$ to $100000 \mathrm{~nm}$. In the coal internal, there is extremely developed micro porosity. Specific surface area, pore volume, pore model of coal and distribution of pore size can be used to characterize the properties of the pore structure. Therefore, the study of coal's microscopic pore structure characteristics is very important. Certain theoretical basis can be provided for the study of coal's some mechanism, such as gas adsorption, desorption and seepage and so on. In recent years, many scholars at home and abroad have done a lot of research on pore characteristics of coal sample and obtained certain achievement by using low-temperature nitrogen adsorption method and the mercury intrusion method[4-6].Based on the above analysis, the author measure adsorption isotherm of different coal particle, calculate and analyze some micro-structure characteristic parameters of coal, such as the coal particle specific surface area, entrance, and the average pore diameter by using American Quantachrome Autosorb iQ specific surface area and pore size analyzer based on the principle of cryogenic liquid adsorption.

\section{Experimental Section}

\section{Experimental Apparatus}

As shown in Fig. 1, the American Quantachrome Autosorb - iQ specific surface area and pore 
size analyzer is used in the experiment. Two degassing stations are built in the Autosorb-iQ, at the same time, samples can be analyzed and prepared. Langmuir specific surface area, single point and multi-point BET specific surface area, $\mathrm{DH}$, the $\mathrm{BJH}$ pore size distribution, pore size distribution, DR micropore area, average pore diameter, total pore volume and other physical parameters can be tested and calculated in the instrument.

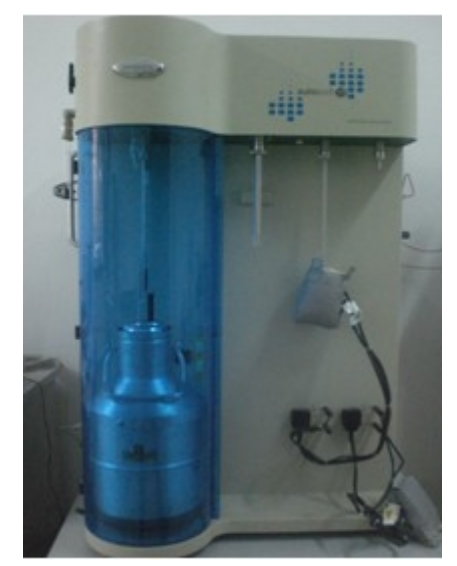

Fig.1 Autosorb-iQ surface area and pore size analyzer from Quantachrome U.S

\section{Coal Sample Preparation}

The samples of 9\# coal seam working face in shanxi phoenix mountain ore are collected in the experiment. Five different particle size of coal samples' particles are prepared into10 20, 20 40, $40 \sim 60,60 \sim 80,80 \sim 100$ mesh in the laboratory, as shown in Table 1.

\section{Experimental Procedure}

BET test is adopted in this experiment, using high purity $\mathrm{N}_{2}$ purity $(99.999 \%)$ as the adsorbate under the condition of liquid nitrogen boiling point temperature $77.2 \mathrm{~K}$. And $\mathrm{N}_{2}$ adsorption volume under different pressure is measured. The relative pressure is set at $0 \sim 1$. Prior to pore structure analysis, the coal samples were degassed under vacuum. The data collected was processed automatically by the software.

Table 1. Coal Sample Parameters

\begin{tabular}{cccc}
\hline Number & $\begin{array}{c}\text { Coal particle } \\
\text { mesh }\end{array}$ & $\begin{array}{c}\text { Particle size of the } \\
\text { corresponding(um) }\end{array}$ & $\begin{array}{c}\text { Average particle } \\
\text { size }(\mathrm{mm})\end{array}$ \\
\hline 1 & $10-20$ & $840-2000$ & 1.42 \\
2 & $20-40$ & $420-840$ & 0.63 \\
3 & $40-60$ & $250-420$ & 0.335 \\
4 & $60-80$ & $178-250$ & 0.214 \\
5 & $80-100$ & $150-178$ & 0.164 \\
\hline
\end{tabular}

\section{$\mathbf{N}_{2}$ Adsorption Isotherms}

Fig. 2 shows that adsorption isotherm of different particle size of coal sample show a similar trend for the $\mathrm{N}_{2}$ adsorption. $\mathrm{N}_{2}$ adsorption quantity increases with the increase of relative pressure $\mathrm{p} / \mathrm{p}_{0}$, and with the decrease of the particle size, coal grain of $\mathrm{N}_{2}$ adsorption quantity increases.Against the types of adsorption isotherm divided by international union of pure and applied chemistry (IUPAC), the adsorption isotherm of coal grains adopted in this experiment are type $\square$ isotherm categories. The first half of the curve is rising slowly and is in the shape of convex upward, which shows that this stage is a phase from monolayer adsorption transition to multilayer adsorption in the inside of a coal particles. And in the second half, the curve rises gently. When the 
relative pressure reaches 0.95 , the curve begins to rise sharply. This shows that the capillary condensation phenomena occurred in the larger hole of coal body, causing a sharp increase of adsorption.

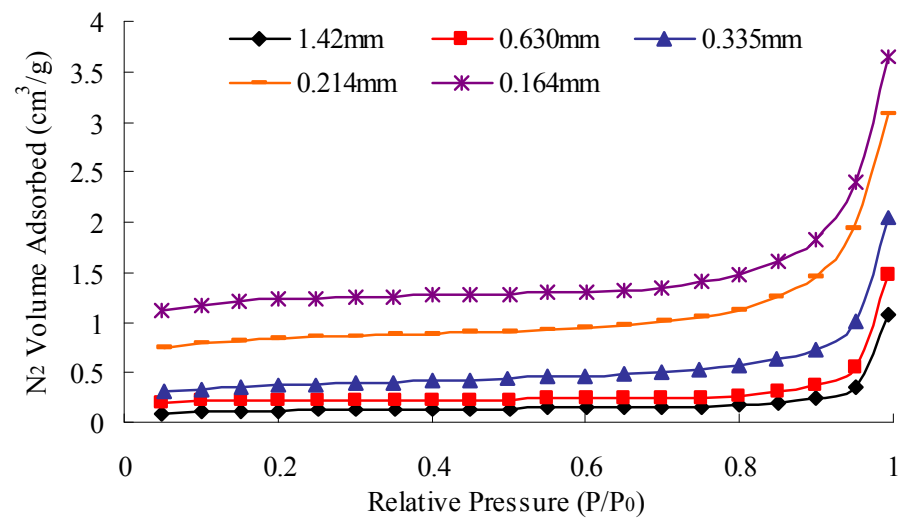

Fig. 2. $\mathrm{N}_{2}$ adsorption isotherms

\section{Experimental Results}

\section{Evaluation of Pore Characteristics}

Specific surface area, entrance and the average pore size are used to characterize of coal granule structure main parameters and they have a different degree of influence on its adsorption amount of adsorbent.According to the characteristics of the sample sorption isotherm, specific surface area of the adsorbent is calculated by BET method, and the total entrance and pore size distribution of the sample is calculated by Kelvin equation[7].

The BET model is

$$
\frac{p}{q\left(p_{0}-p\right)}=\frac{1}{q_{m} c}\left[1+\frac{p}{p_{0}}(c-1)\right]
$$

where, $q_{m}$ is the weight of adsorbate constituting a monolayer of surface coverage, and $q$ is the weight of gas adsorbed at a relative pressure $\left(P / P_{0}\right)$. The term $\mathrm{C}$ (the BET constant) is related to the energy of adsorption in the first adsorbed layer and consequently its value is an indication of the magnitude of the adsorbent/adsorbate interactions.

The Kelvin equation is described as:

$\ln \frac{p}{p_{0}}=\frac{2 \gamma \bar{V}}{r R T} \cos \theta$

In the above equation: $r$ is expressed as pore range and given by analysis software automatically, $\gamma$ is expressed as the surface tension of liquid nitrogen, $T$ is represented as Absolute temperature, $\bar{V}$ is represented as liquid molar volume, $R$ is represented as general gas constant, $\theta$ is represented as adsorption angle and is value to 0 for liquid adsorption under high pressure.

\section{Results and Discussion}

Micro-structure characteristic parameters of coal with different particle size of grainare are calculated, the results as shown in Table 2. It shows that within a certain particle size range, with the decrease of the coal particle size, specific surface area grains and total entrance increase gradually, the corresponding average pore diameter is reduced. The results and the study of literature are consistent [8]. The relationship between specific surface area and the coal particle size is shown in Fig. 3. It shows that specific surface area decreases with the increase of coal granularity within a 
certain particle size. The greater the specific surface area is, the more fully gas-solid contact, which can produce beneficial effect on the reaction gas adsorption, desorption and diffusion and so on. The relationship between overall pore volume and the coal abrasive is shown in Fig. 4. It shows that total pore volume decreases with the increase of coal granularity within a certain particle size. The relationship between average pore diameter and coal particle size is shown in Fig. 5, which shows that coal grain of average pore diameter increases with the increase of coal granularity within a certain particle size.

Tableb2. Experimental results of coal particle structure parameters

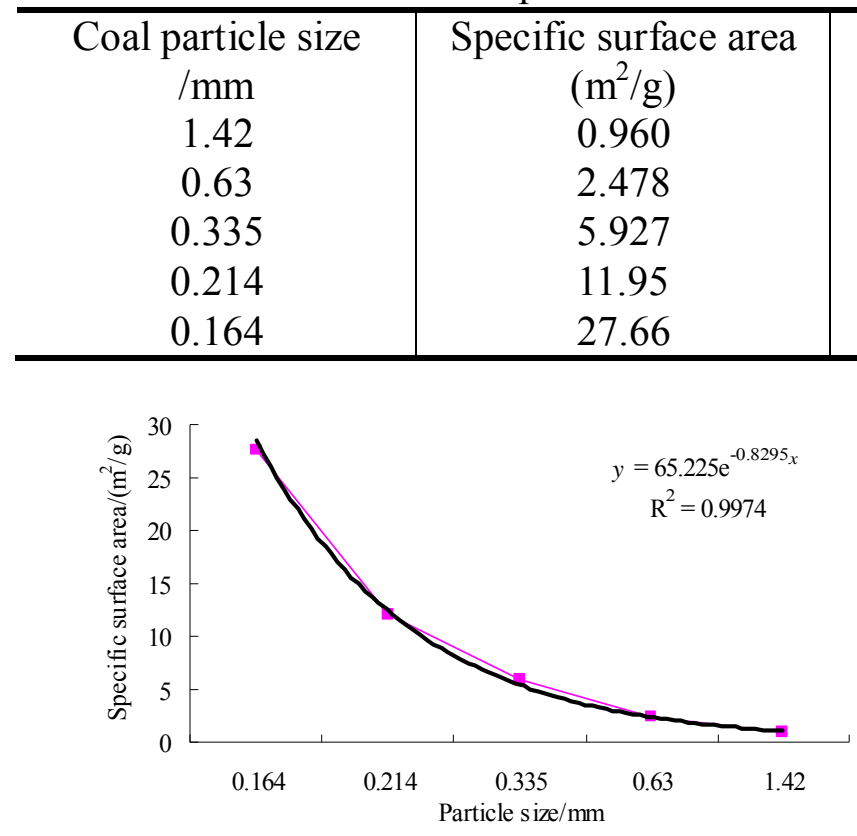

Fig.3 Relation diagram between surface area and coal particle size
Total pore volume $\left(\mathrm{cm}^{3} / \mathrm{g}\right)$

$2.126 \times 10^{-2}$

$9.752 \times 10^{-3}$

$5.029 \times 10^{-3}$

$2.130 \times 10^{-3}$

$9.981 \times 10^{-4}$
Average pore size (nm)

4.159

3.438

3.394

3.264

3.074

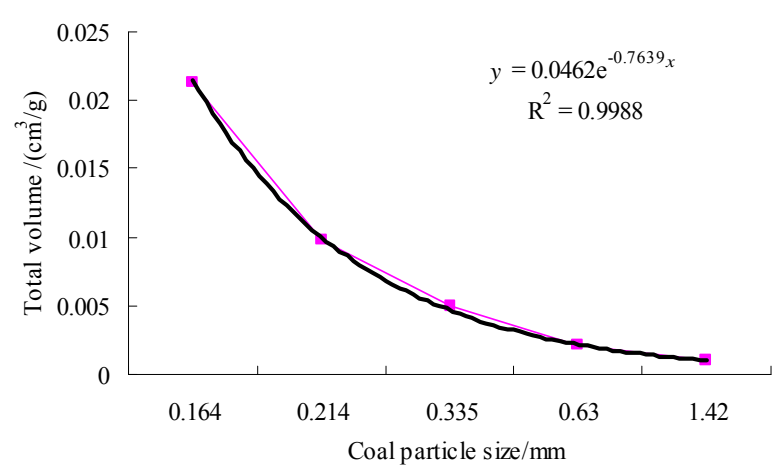

Fig.4 Relation diagram between total pore volume and coal particle size

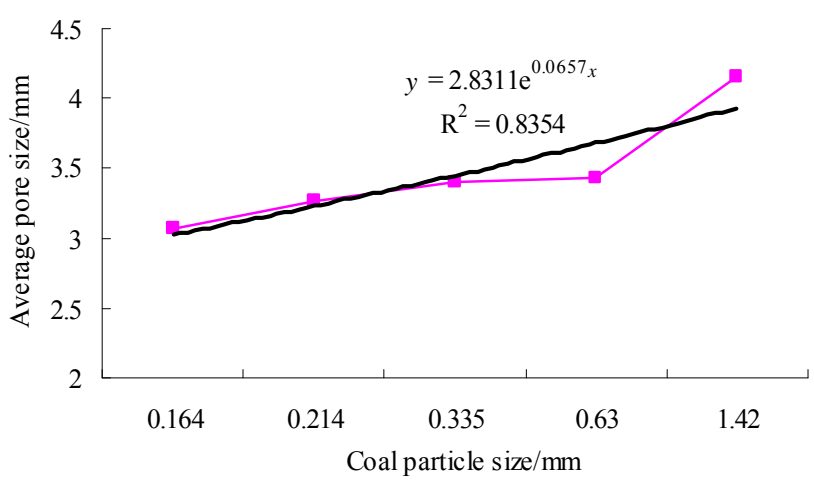

Fig. 5 Relation diagram between average pore size and coal particle size

\section{Conclusion}

1) Based on the principle of low temperature liquid nitrogen adsorption and by using contador Quantachrome Autosorb - iQ specific surface area and pore size analyzer, adsorption isotherm of different particle size of coal particles is studied. The results show that the adsorption isotherm of different particle size of coal particles present a similar change trend. $\mathrm{N}_{2}$ adsorption quantity increases with the increase of relative pressure, and coal grain of $\mathrm{N}_{2}$ adsorption quantity increases with the decrease of the coal granularity. 
2) Coal particle micro-structure parameters such as specific surface area, entrance, and the average pore size) are calculated and analyzed. The results show that at a certain particle size range, specific surface area of the coal grain and overall pore volume show a decrease trend with the increase of coal granularity, while the average pore diameter show a trend of increase.

\section{Reference}

[1] B.F. Yu. Technology handbook of prevention and control and using of gas disaster in coal mine. Beijing: coal industry publishing house, 2000.

[2] L. Zhang, X.Q. He, B.S. Nie. Study on the process of coal absorbing gas, Journal of mining safety and environmental protection, 27(2000) 1-4

[3] L.J. Xu, X.F. Xian, C.L. Liu. Research of structure characteristics of highlight area coal pore, The mining safety and environmental protection. 26(1991)25-27.

[4] D.S. Xing, W.P. Yan. Analysis to pore structure of typical semi-cokes by mercury porosimetry, Journal of North China Electric Power University(Natural Science Edition). 34(2007)57-63

[5] W.P. Xiang, X.Z. Song, L.W. Zhong. Experiment of coal pore characteristics of different coal body structure and its effect on gas outburst based on the low temperature liquid nitrogen, Journal of China Coal Society. 36(2011)609-614

[6] L.L. Qi, Z.F. Wang, H.M. Yang. Study on porosity of coal samples based on low temperature nitrogen adsorption method and mercury porosimetry, Coal Science and Technology. 40(2012)36-39

[7] S. Brunauer, P. Emmett, H. Teller. Adsorption of gasesin multimolecular layers. Journal of the AmericanChemistry Society, 1938( 60):309 - 319.

[8] G.P. Ren, C.Q. Zhang, X.M. Jiang. Analysis of surface area and pore structure of Datong coal, Journal of Combustion Science and Technology, 13(2007)265-268 